\title{
PERCEPÇÃO DA DOENÇA E DE RISCO \\ NA OBESIDADE: REVISÃO SISTEMÁTICA
}

ILLNESS AND RISK PERCEPTION IN OBESITY: A SYSTEMATIC REVIEW

Recibido: 02 de septiembre de 2019 | Aceptado: 05 de junio de 2020

\author{
FABIANA BRUM SCHAKAROWSKI 1, CLAUDIO CORÁ MOTTIN 2, ELISA KERN DE CASTRO 3
}

1., 3. UNIVERSIDADE DO VALE DO RIO DOS SINOS (UNISINOS), Rio Grande do Sul, Brasil

2. PONTIFÍCIA UNIVERSIDADE CATÓLICA DO RIO GRANDE DO SUL (PUCRS), Rio Grande do Sul, Brasil

\begin{abstract}
BACKGROUND: Obesity has been described as a chronic, multifactorial and evolutionary disease, putting the lives of individuals at risk. OBJETIVE: The objective of this study was to perform a systematic review on the perception of the disease and the risk in obesity between January 2004 and January 2018. METHOD: The Lilacs, SciELO, MEDLINE, PubMed, PsycINFO and Web of Science databases were searched of Science, studies investigating the perception of the disease and the risk of obesity based on behavioral and social theories that help to understand why adults engage in risky behaviors. RESULTS: From 577 references, seven studies met the inclusion criteria. It has been found that obese individuals have difficulty understanding their disease as a chronic disease that requires continuous efforts to control it. The evaluation of the perception of risk of obesity did not promote individual preventive actions of the disease nor the adoption of appropriate behaviors to improve health status. CONCLUSION: Risk perception is a construct that assists in the understanding of obesity and can subsidize practices of behavior change intervention in patients.
\end{abstract}

KEY WORDS: Risk perception, IIIness perception, Illness beliefs, Obesity.

\section{CÓMO CITAR / HOW TO CITE}

Brum Schakarowski, F., Corá Mottin, C., \& Kern De Castro, E. (2020). Percepção da doença e de risco na obesidade: revisão sistemática. Salud \& Sociedad, 11, e3737. doi: 10.22199/issn.0718-7475-20200003 
A obesidade tem sido descrita como uma doença crônica, multifatorial e de caráter evolutivo (Noria \& Grantcharov, 2013; Yamada-Goto, Katsuura, \& Nakao, 2013), colocando em risco a vida dos indivíduos (Falba \& Busch, 2005). Embora, uma percepção de risco mais acurada esteja relacionada às mudanças nos padrões de gestão da doença, muitos indivíduos apresentam dificuldades para reavaliar a percepção individual sobre sua saúde (Bonsaksen, Lerdal, \& Fagermoen, 2015). Nesse sentido, diferentes modelos teóricos têm sido utilizados com ênfase em resultados mais consistentes para a redução de fatores de risco, melhora de resposta emocional à doença e nas crenças sobre 0 comportamento de indivíduos obesos. Dentre esses modelos teóricos, destacam-se - Modelo de Autorregulação em Saúde (Leventhal, et al., 1984), a Teoria da Ação Racional (Ajzen \& Fishbein, 1980; Fishbein \& Ajzen, 1975), 0 Modelo de Protótipo de Disposição (Rivis, et al. 2006) a Teoria Social Cognitiva (Bandura, 1977; 1991), e o Modelo Transteórico de Mudança de Comportamento (Prochaska \& DiClemente, 1982).

O Modelo de Autorregulação em Saúde, também denominado Modelo do Senso Comum (MSC), enfatiza que o indivíduo possui um sistema autorregulador que integra as representações ou crenças sobre a doença, sendo usado para gerenciar eventuais ameaças à sua saúde (Leventhal et al., 1984). Essas representações cognitivas de ameaça da doença incidem sobre as crenças individuais, tendo reflexo no seu enfrentamento (Figueiras \& Weinman, 2003; Leventhal et al., 1984). A percepção da doença pode ser identificada através de sete domínios: (a) identidade: significado e sintomas atribuídos à doença; (b) causas: crenças sobre o desenvolvimento da doença; (c) duração: percepção aguda, crônica ou cíclica da doença; (d) consequências: impacto da doença sobre a vida do indivíduo; (e) controle: cura ou tratamento da doença através da autogestão comportamental; (f) Coerência: compreen- são individual e $(\mathrm{g})$ representação emocional: impacto emocional da doença (Cameron \& Leventhal, 2003; Leventhal et al., 1984; Leventhal, et al., 2003; Crouch \& Leventhal, 1997).

A Teoria da Ação Racional (TRA) tem como foco a identificação de fatores preditores do comportamento consciente intencional. Presume que as atitudes dos indivíduos em relação à sua saúde/doença estão determinadas por três fatores: (a) cognitivo: as crenças são atribuídas às consequências de sua conduta, (b) social: a execução ou não do comportamento pode ser incentivado pela pressão socialmente exercida, e (c) comportamental: reações dos indivíduos (Ajzen \& Fishbein, 1980; Fishbein \& Ajzen, 1975). Esse modelo considera que os indivíduos devem se comportar de maneira racional, ponderando perdas e ganhos em relação ao controle volitivo (Ajzen, 1985; Ajzen, 1991). As intenções, as atitudes e as normas sociais devem permear as ações individuais, para favorecer 0 comportamento e manutenção saudáveis. Sendo assim, a intenção do indivíduo em relação ao seu comportamento em saúde será maior quanto mais positiva a avaliação sobre suas atitudes e as normas subjetivas (Ajzen, 1991; Ajzen \& Fishbein, 1980).

O Modelo de Protótipo de Disposição (PWM) incorpora a Teoria da Ação Racional (TRA) e reação social, sustentando que as imagens ou protótipos têm impacto significativo sobre as decisões do indivíduo de engajar-se em comportamentos de proteção à saúde (Rivis, et al., 2006). Essas decisões devem abranger um comportamento preditor, influenciado pela imagem de risco social (avaliação do protótipo) e de similaridade da imagem a si mesmo (semelhança de protótipo). Enfatiza, ainda, que 0 comportamento anterior influencia as atitudes atuais e, que a vulnerabilidade percebida $e$ as normas subjetivas corroboram com o comportamento de intenções para prevenção de riscos. Sendo assim, quanto maior percepção da similaridade com 0 protótipo, maior será 
inclinação do indivíduo para se envolver na prevenção de risco à saúde.

A Teoria Social Cognitiva (Bandura, 1977), considera a intencionalidade e a previsão de possíveis resultados como fatores que regulam a representação cognitiva dos indivíduos para o planejamento de estratégias. Nessa teoria, a autoeficácia está associada às crenças individuais que influenciam as atitudes dos indivíduos (Bandura, 1991). Esse modelo aponta para a capacidade de autorregulação do comportamento do indivíduo, através do aprendizado e análise crítica sobre determinadas experiências. Assim, a aquisição de novas habilidades pode ressignificar cognitivamente a percepção, de forma mais realista, para o desenvolvimento de um comportamento mais saudável. 0 modelo tem sido amplamente utilizado na saúde (Lerdal, et al., 2011; Simpson, Lekwuwa, \& Crawford, 2013).

O Modelo Transteórico de Mudança de Comportamento (MTT) foca na premissa de que a mudança comportamental ocorre ao longo de um processo no qual os indivíduos passam por diversos níveis de motivação para mudança (estágios) (Prochaska, 1979, Prochaska \& Velicer, 1997). Esses estágios correspondem às atividades implícitas ou explícitas nas quais os indivíduos conseguem alterar suas atitudes, buscando a estabilização do comportamento saudável. Os estágios motivacionais são: précontemplação, contemplação, preparação, ação e etapas de manutenção (terminação e recaída). Criado inicialmente para compreender comportamentos aditivos (Prochaska, Diclemente, \& Norcross, 1992), esse modelo ampliou sua aplicação para a saúde em geral (Diclemente, et al., 2004; Johnson et al., 2006; Johnson, et al. 2013; Mastellos, et al., 2014). Em intervenções com indivíduos obesos para perda de peso, - MTT mostra que os estágios de mudança são inconsistentes para além de doze meses (Johnson, et al., 2006; Prochaska, et al., 2008; Wee, et al., 2005). Isso mostra que no manejo da obesidade, nem sempre os indivíduos estão dispostos a mudar seus comportamentos mesmo sabendo dos riscos inerentes à sua doença. A mudança de comportamento tem que ser voluntária para ser sustentável (Mastellos et al., 2014).

Com relação à percepção de risco, esse constructo é essencial para aferir julgamentos imprecisos sobre a doença (Slovic, et al., 2004). O nível de julgamento do risco por um indivíduo sobre sua doença inclui o conhecimento, sentido de controle e características relacionados ao próprio risco (Slovic, 1987; Siegrist \& Cvetkovich, 2000). A percepção de risco pode ser avaliada como a probabilidade percebida da ocorrência do risco, abrangendo o grau de preocupação ou medo associado (Sjöberg, 1998). A percepção de gravidade da doença, a representação emocional e as estratégias de enfrentamento podem se modificar ao longo do tempo na medida em que novas informações sobre a doença são introjetadas pelo indivíduo (French, et al., 2000; Furze, et al.,2002).

Assim, muitos fatores contribuem para 0 processo de mudança comportamental no que tange à doenca de obesidade. Frente a isso torna-se relevante examinar os modelos teóricos que têm abordado a percepção da doença e de risco em indivíduos obesos. 0 desenvolvimento desse campo de investigação poderá sumarizar os achados existentes nessa área e elucidar possíveis lacunas a respeito do conhecimento na temática, contribuindo com ações em saúde que favoreçam a promoção, prevenção e intervenção na doença de obesidade.

\section{MÉTODO}

A revisão sistemática foi conduzida a partir das recomendações propostas no guia Preferred Reporting Items for Systematic Reviews and Meta-Analyses (PRISMA) (Moher, et al., 2009). Na busca dos artigos, foram empregados os seguintes descritores - em inglês: "risk perception" OR "illness perception" OR "illness representation" OR "illness beliefs" AND "obesity", nas bases de 
dados Lilacs, SciELO, MEDLINE, PubMed, PsycINFO e Web of Science. A pesquisa incluiu o período de janeiro de 2004 a janeiro de 2018. 0 critério de seleção das bases de dados foi em virtude de serem referência na busca de material científico na área da saúde.

Os critérios de inclusão dos artigos no presente estudo foram: (a) amostra de adultos com obesidade; (b) idade igual ou superior a 18 anos; (c) objeto de estudo ser a percepção da doença e de risco da obesidade. Excluiu-se da revisão: (a) estudos cuja avaliação eram doenças crônicas não transmissíveis e a obesidade era uma variável secundária (câncer de mama, diabetes mellitus tipo 2, trombose, doenças cardiovasculares); (b) indicadores de saúde em que a obesidade também era uma variável secundária (sintomas na menopausa, prática regular de atividade física, prevenção de erros médicos em comportamentos de precaução).

Os estudos foram analisados seguindo os critérios do Protocolo de Pesquisa PRISMA. A seleção dos títulos e resumos obedeceu, rigorosamente, aos critérios de inclusão e exclusão. Foram observados a mensuração dos desfechos de interesse, verificação da acurácia dos resultados, determinação dos critérios metodológicos e tipos de estudos que investigavam a percepção da doença $e$ de risco da obesidade. Em seguida, foram marcados os estudos repetidos dentro das bases e entre elas. Posteriormente, realizouse um refinamento para selecionar os estudos referentes à temática abordada por meio da avaliação de texto completo para determinar sua elegibilidade.

\section{RESULTADOS}

A busca realizada nas bases de dados resultou em 577 artigos, sendo que 54 artigos estavam duplicados nas bases e entre elas. Após a triagem dos títulos e resumos dos 577 artigos, foram excluídos 566 estudos, restando onze artigos (Blixen, Singh, \& Thacker, 2006; Bonsaksen, et al.,
2012; Bonsaksen, Fagermoen, \& Lerdal, 2015; Bonsaksen, Lerdal, \& Fagermoen, 2015; Jumean et al., 2012; Ogden \& Sidhu, 2006; Okop, et al., 2016; Prasad et al., 2014; Radtke, et al., 2014; Teede, et al., 2010; Winter, \& Wuppermann, 2014), e seis artigos duplicados (Hilbert et al., 2009; Juszczyk, et al., 2014; Kaptein et al., 2007; Park, et al., 2011; Parschau et al., 2014; Peters, et al., 2006). No total, 23 estudos completos restaram para avaliar a elegibilidade. Após leitura na íntegra dos 23 estudos, foram excluídos sete artigos com foco em doenças crônicas não transmissíveis em que a obesidade era uma variável secundária e nove artigos com indicadores de saúde em que a obesidade era uma variável secundária. Resultaram sete estudos sobre percepção da doença e de risco na obesidade, publicados entre 2006 e 2016, que preencheram os critérios de inclusão. $A$ Figura 1 representa o fluxograma de pesquisa e seleção dos estudos da revisão sistemática.

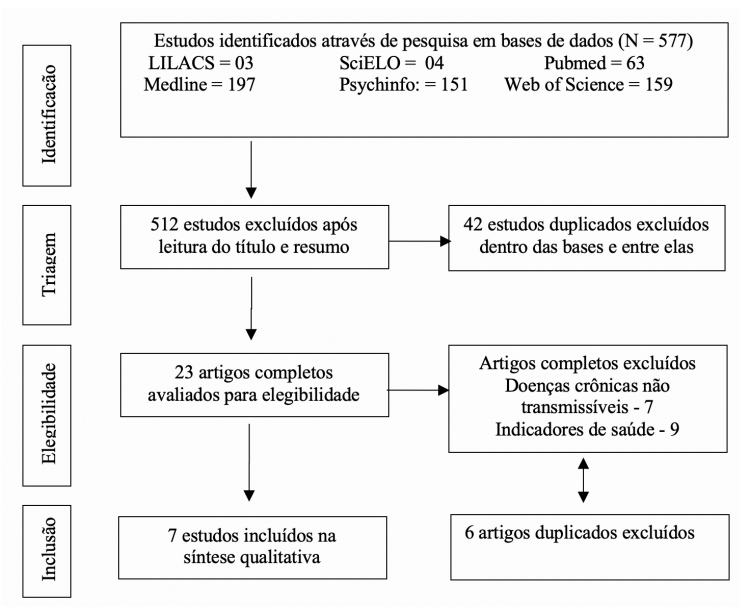

FIGURA 1.

Fluxograma de identificação e seleção dos artigos para revisão sistemática sobre percepção de risco e percepção da doença de obesidade.

\section{Características dos estudos}

Nos estudos selecionados, foram avaliados parâmetros clínicos e metabólicos (hipertensão, artrite, doenças cardiovasculares, diabetes mellitus tipo 2 , acidente vascular cerebral, dentre outras), atividade física e comportamento alimentar. 
As pesquisas foram realizadas nos seguintes países: Noruega (três), EUA (dois), Inglaterra (um) África do Sul (um). A caracterização dos artigos quanto aos objetivos enfatizou dois focos principais: (a) implicações da percepção da doença e estratégias de enfrentamento nas mudanças de comportamento ao longo do tempo (Bonsaksen, Fagermoen, \& Lerdal, 2015; Bonsaksen, Lerdal, \& Fagermoen, 2015; Bonsaksen et al., 2012; Okop, et al., 2016); e (b) implicações da percepção de risco, comorbidades associadas e processo de tomada de decisão (Jumean et al., 2012; Ogden \& Sidhu, 2006; Prasad et al., 2014). No que se referem aos instrumentos, três pesquisas utilizaram 0 questionário de percepção de doença (Illness Perception Questionnaire - IPQ) para mensurar a percepção da doença (Bonsaksen et al., 2012; Bonsaksen, Fagermoen, \& Lerdal,
2015; Bonsaksen, Lerdal, \& Fagermoen, 2015). O apoio social foi medido com a questão aberta: - Eu acho que tenho apoio suficiente das pessoas com as quais tenho uma relação mais próxima? (Bonsaksen, Lerdal, \& Fagermon, 2015). A percepção de risco para obesidade foi avaliada através de intervenção educativa, com questionário em formato likert, entrevistas semi-estruturadas e grupos focais (Jumean et al., 2012, Ogden \& Sidhu, 2006; (Okop, et al., 2016; Prasad et al., 2014). As intervenções educativas relacionadas à percepção de risco foram enfatizadas por motivar os participantes a assumirem um estilo de vida mais saudável (Jumean et al., 2012; Ogden \& Sidhu, 2006). A Tabela 1 contém as principais características dos sete estudos selecionados, que incluíram um total de 948 participantes e cujas pesquisas foram publicadas entre 2006 e 2016.

TABELA 1.

Estudos Sobre Percepção de Risco e Percepção da Doença e Obesidade.

\begin{tabular}{|c|c|c|c|c|}
\hline Estudo & Base teórica & Objetivo/Intervenção & Amostra & Resultados * \\
\hline $\begin{array}{l}\text { Bonsaksen, } \\
\text { Fagermoen, \& } \\
\text { Lerdal (2015) } \\
\text { Oslo, Noruega }\end{array}$ & $\begin{array}{l}\text { Teoria Social } \\
\text { Cognitiva } \\
\text { Modelo do } \\
\text { Senso } \\
\text { Comum }\end{array}$ & $\begin{array}{l}\text { Explorar variáveis de trabalho, } \\
\text { atividade física, percepções da } \\
\text { doença e autoeficácia em relação à } \\
\text { autoestima. Escala de Rosenberg } \\
\text { (Rosenberg, 1965) Escala de } \\
\text { autoeficácia (Schwarzer \& } \\
\text { Jerusalém, 1995) Questionário de } \\
\text { percepção da doença (Broadbent et } \\
\text { al., 2006) Inquérito HUNT-2 } \\
\text { (Holmen et al., 2003). }\end{array}$ & $\begin{array}{l}\text { Pacientes obesos } \\
\text { mórbidos }(\mathrm{N}=134) \\
\text { Pacientes com } \\
\text { doença pulmonar } \\
\text { obstrutiva crônica } \\
(\mathrm{N}=89)\end{array}$ & $\begin{array}{l}\text { Indivíduos obesos apresentaram maior } \\
\text { percepção de consequências da doença, } \\
\text { menor controle pessoal e maior } \\
\text { preocupação que pacientes com DPOC. } \\
\text { Quanto mais autoestima, mais curta foi a } \\
\text { percepção de duração da doença. A } \\
\text { autoestima foi maior nos indivíduos com } \\
\text { DPOC, porém com percepção de menor } \\
\text { controle de tratamento. }\end{array}$ \\
\hline
\end{tabular}

\begin{tabular}{|c|c|c|}
\hline $\begin{array}{l}\text { Bonsaksen, } \\
\text { Lerdal, \& } \\
\text { Fagermoen } \\
\text { (2015) } \\
\text { Oslo, Noruega }\end{array}$ & $\begin{array}{l}\text { Modelo do } \\
\text { Senso } \\
\text { Comum }\end{array}$ & $\begin{array}{l}\text { Avaliar a percepção da doenca e } \\
\text { estratégias de enfrentamento em } \\
\text { relação à capacidade de detectar } \\
\text { mudanças na saúde. Questionário } \\
\text { de percepção da doença (Broadbent } \\
\text { et al., 2006) aplicado cinco vezes ao } \\
\text { longo de um ano. }\end{array}$ \\
\hline $\begin{array}{l}\text { Okop, } \\
\text { Mukumbang, } \\
\text { Mathole, Levitt } \\
\text { \& Puoane, } \\
\text { (2016) } \\
\text { Langa, } \\
\text { África do Sul }\end{array}$ & $\begin{array}{l}\text { Modelo } \\
\text { Protótipo de } \\
\text { Disposição } \\
\text { Teoria da } \\
\text { Ação } \\
\text { Racional }\end{array}$ & $\begin{array}{l}\text { Explorar as percepções da imagem } \\
\text { corporal, percepção de risco da } \\
\text { obesidade e disposição para perder } \\
\text { peso entre adultos de comunidade } \\
\text { com recursos limitados, para fins de } \\
\text { intervenções para a prevenção da } \\
\text { obesidade. Oito discussões, em } \\
\text { Grupos focais, entre agosto de } 2014 \\
\text { e fevereiro de } 2015 \text {. }\end{array}$ \\
\hline
\end{tabular}

Pacientes obesos mórbidos ( $\mathrm{N}=53)$ Pacientes com doença pulmonar obstrutiva crônica $(\mathrm{N}=52)$

Mulheres ( $\mathrm{N}=36)$ Homens $(\mathrm{N}=42)$ Categorizados pelo índice de massa corpórea e separados por sexo
Ao longo do tempo, indivíduos obesos perceberam sua doença como menos ameaçadora e com maior controle pessoal. Entre os indivíduos com DPOC houve maior controle pessoal.

Participantes obesos que experimentaram condições de doença crônica tiveram maior percepção de risco de obesidade e de doença cardiovascular. A crença de que excesso de peso é normal, e não uma doença, influenciaram negativamente a vontade dos participantes para perder peso. 0 estudo revelou que as mulheres com excesso de peso não se perceberam em risco de obesidade 


\begin{tabular}{|c|c|c|c|c|}
\hline Estudo & Base teórica & Objetivo/Intervenção & Amostra & Resultados * \\
\hline $\begin{array}{l}\text { Jumean et al. } \\
\text { (2012) } \\
\text { EUA }\end{array}$ & $\begin{array}{l}\text { Modelo } \\
\text { Transteórico }\end{array}$ & $\begin{array}{l}\text { Avaliar o efeito do diagnóstico de } \\
\text { síndrome metabólica sobre a } \\
\text { percepção de risco e as mudanças } \\
\text { de comportamento na saúde. } \\
\text { Pacientes receberam material de } \\
\text { intervenção educativo (cartilha e } \\
\text { vídeos) sobre síndrome metabólica } \\
\text { e fatores de risco para a saúde. }\end{array}$ & $\begin{array}{l}\text { Pacientes com } \\
\text { síndrome metabólica } \\
\text { ou fator de risco } \\
\text { cardiovascular } \\
(\mathrm{N}=74)\end{array}$ & $\begin{array}{l}\text { Indivíduos obesos, ao receber o } \\
\text { diagnóstico de síndrome metabólica } \\
\text { associada com intervenção educativa, } \\
\text { aumentaram a percepção do risco } \\
\text { cardiovascular, a preocupação com a } \\
\text { saúde e motivação para estilo de vida } \\
\text { mais saudável. }\end{array}$ \\
\hline
\end{tabular}

\begin{tabular}{|c|c|c|c|c|}
\hline $\begin{array}{l}\text { Bonsaksen, } \\
\text { Lerdal, \& } \\
\text { Fagermoen } \\
\text { (2012) } \\
\text { Oslo, Noruega }\end{array}$ & $\begin{array}{l}\text { Teoria Social } \\
\text { Cognitiva } \\
\text { Modelo do } \\
\text { Senso } \\
\text { Comum }\end{array}$ & $\begin{array}{l}\text { Examinar apoio social, atividade } \\
\text { física e percepção da doença em } \\
\text { relação a autoeficácia. Escala de } \\
\text { Percepção de autoeficácia } \\
\text { (Schwarzer \& Jerusalém, 1995) } \\
\text { Inquérito HUNT-2 (Holmen et al., } \\
\text { 2003) } \\
\text { Questionário de Percepção da } \\
\text { Doença (Broadbent, et al., 2006) }\end{array}$ & $\begin{array}{l}\text { Pacientes obesos } \\
\text { mórbidos }(\mathrm{N}=134) \\
\text { Pacientes com } \\
\text { doença pulmonar } \\
\text { obstrutiva crônica } \\
(\mathrm{N}=86)\end{array}$ & $\begin{array}{l}\text { Indivíduos obesos perceberam mais } \\
\text { consequências da doença, menor } \\
\text { controle pessoal e maior preocupação } \\
\text { que individuos com DPOC. Em contraste, } \\
\text { indivíduos com DPOC perceberam menor } \\
\text { controle do tratamento, que foi } \\
\text { relacionado à maior autoeficácia. }\end{array}$ \\
\hline $\begin{array}{l}\text { Ogden \& } \\
\text { Sidhu (2006) } \\
\text { Londres, } \\
\text { Inglaterra }\end{array}$ & $\begin{array}{l}\text { Modelo do } \\
\text { Senso } \\
\text { Comum }\end{array}$ & $\begin{array}{l}\text { Examinar as crenças para uso de } \\
\text { medicação para emagrecimento, } \\
\text { como um meio para explorar a } \\
\text { adesão e mudança de } \\
\text { comportamento. Entrevistas } \\
\text { semi-estrutruradas com análise } \\
\text { fenomenológica (Smith \& Osborn, } \\
\text { 2003). }\end{array}$ & $\begin{array}{l}\text { Pacientes que } \\
\text { tomaram Orlistat por } \\
\text { pelo menos dois } \\
\text { anos }(\mathrm{N}=12)\end{array}$ & $\begin{array}{l}\text { As crenças sobre as causas da obesidade } \\
\text { variaram de fatores externos a controle } \\
\text { pessoal. Os efeitos colaterais do } \\
\text { medicamento podem mudar as crenças } \\
\text { dos indivíduos obesos sobre as causas } \\
\text { da obesidade, motivando-os a mudar o } \\
\text { comportamento alimentar para perda de } \\
\text { peso. }\end{array}$ \\
\hline $\begin{array}{l}\text { Prasad et al. } \\
\text { (2014) } \\
\text { EUA }\end{array}$ & $\begin{array}{l}\text { Teoria da } \\
\text { Ação } \\
\text { Racional }\end{array}$ & $\begin{array}{l}\text { Examinar o risco cardiovascular e } \\
\text { percepção de risco cirúrgico. Risco } \\
\text { para obesidade e risco cirúrgico } \\
\text { foram avaliados num questionário } \\
\text { em formato Likert. Para o Risco } \\
\text { cardiovascular foi usado Escore de } \\
\text { Risco de Framingham (Wilson et al., } \\
\text { 1998). }\end{array}$ & $\begin{array}{l}\text { Pacientes obesos em } \\
\text { tratamento clínico } \\
(\mathrm{N}=148) \text { Pacientes } \\
\text { obesos submetidos à } \\
\text { cirurgia bariátrica } \\
(\mathrm{N}=88)\end{array}$ & $\begin{array}{l}\text { Indivíduos obesos submetidos à cirurgia } \\
\text { bariátrica são mais propensos do que os } \\
\text { indivíduos tratados clinicamente para } \\
\text { perceber a obesidade como um maior } \\
\text { risco para a sua saúde. }\end{array}$ \\
\hline
\end{tabular}

\section{DISCUSSÃO}

A presente revisão sistemática mostrou que diferentes modelos teóricos têm sido utilizados para investigar a percepção de risco e percepção da doença da obesidade. Os modelos apresentados abrangem os comportamentos dos indivíduos frente à doença, incluindo os processos que envolvem a adoção de mudanças e estratégias de enfrentamento relacionadas ao estilo de vida. Os resultados serão discutidos a partir das dimensões abordadas em cada modelo teórico, que influenciam a condição da gestão da doença.

O Modelo de Autorregulação (MSC) em saúde tem sido amplamente empregado para compreender a percepção da doença. Com respeito à doença de obesidade, os estudos que utilizaram o MSC demonstraram características específicas que as pessoas percebem sobre essa doença. No que se refere à dimensão Identidade, a percepção de sintomas está relacionada à percepção dos efeitos colaterais da medicação para emagrecer (Ogden \& Sidhu, 2006), ao grau de aderência e à preocupação sobre a doença (Bonsaksen, Lerdal, \& Fagermoen, 2015). Esses dados revelam que as decisões individuais relacionadas às mudanças na saúde parecem ter relação com a quantidade e intensidade dos sintomas da obesidade e dos medicamentos.

Com relação à duração da doença, 0 impacto de receber um diagnóstico de síndrome metabólica pôde aumentar a preocupação com a saúde dos indivíduos obesos e a motivação em direção a um estilo de vida mais saudável (Jumean et al., 2012). Apesar disso, apenas receber o diagnóstico 
não induz alterações comportamentais, já que entre o desejo de mudar e a mudança de comportamento em si existe um desafio considerável na gestão do autocuidado. Corroborando esta ideia, os estudos revisados mostraram que percepção de menor duração da doença é percebida quando ela é vista como menos ameaçadora (Bonsaksen et al., 2012; Bonsaksen, Fagermoen, \& Lerdal, 2015; Bonsaksen, Lerdal, \& Fagermoen, 2015; Jumean et al., 2012; Ogden \& Sidhu, 2006). A percepção de duração curta da obesidade pode indicar uma crença de que a condição de obeso mudará por si só (Bonsaksen, Fagermoen, \& Lerdal, 2015). Por outro lado, mudanças no estilo de vida e tratamentos associados podem levar a uma visão alterada de duração da doença (Bonsaksen, Lerdal, \& Fagermoen, 2015). Portanto, a percepção de duração da doença possivelmente interfere na capacidade de autogestão dos indivíduos e, consequentemente, na autoestima.

Em termos de causas percebidas, cabe ressaltar a relação entre a crença de causas genéticas da obesidade (onde individuo atribui não ter controle próprio) e o controle alimentar (Ogden \& Sidhu, 2006). Os indivíduos com dificuldades para mudar seu comportamento se enquadram dentro de uma atribuição causal da obesidade que tem como foco as questões genéticas. Em contrapartida, os indivíduos que aderiram a uma dieta equilibrada atribuíam as causas da obesidade a questões comportamentais e, portanto, passíveis de modificação. A crença na eficácia do medicamento para emagrecer sugere uma ideação relacionada à eliminação do excesso de peso (Ogden \& Sidhu, 2006). Portanto, é importante identificar a que o indivíduo atribui a causa da sua obesidade para entender que representação ele faz dessa doença e, 0 quanto ele se perceberá implicado na mudança de comportamento. Esse achado traz perspectivas interessantes para 0 psicólogo da saúde trabalhar na mudança de crenças e, consequentemente, do comportamento do indivíduo obeso que necessita mudar seus hábitos cotidianos.
Acerca das consequências, os indivíduos obesos perceberam sua doença como menos ameaçadora ao longo do tratamento (Bonsaksen, Lerdal, \& Fagermoen, 2015). Em outros estudos revisados (Bonsaksen et al., 2012; Bonsaksen, Fagermoen, \& Lerdal, 2015) o apoio social favoreceu o aumento de atividade física com enfrentamento para mudanças no estilo de vida. Isso confirma que a compreensão da doença depende de vários fatores, mas principalmente do estado de saúde percebido e do controle da doença. Ao que tudo indica alguns desses indivíduos subestimam 0 impacto das consequências negativas que a doença pode trazer à sua saúde. A partir desse recorte, é importante estimular nesses indivíduos uma percepção positiva das mudanças para que mantenham comportamentos alinhados com a autogestão e melhora da qualidade de vida.

A percepção de controle da doença foi trabalhada nos estudos revisados através de programas educativos aliados à gestão de estratégias de enfrentamento (Bonsaksen et al., 2012; Bonsaksen, Fagermoen, \& Lerdal, 2015; Bonsaksen, Fagermoen, \& Lerdal, 2015; Ogden \& Sidhu, 2006). Pode-se observar que a aquisição de novos comportamentos diante do tratamento da obesidade influencia a motivação para produzir resultados mais duradouros. Esse parece ser um processo consciente de gestão pessoal para a adoção de estratégias positivas para melhora da qualidade de vida. Entender esse processo implica em propostas de intervenções voltadas para níveis de comportamentos que são multifatoriais, assegurando uma proposta mais integradora da doença de obesidade.

Com relação ao entendimento do individuo sobre a sua doença e percepção de risco, parece que os obesos têm dificuldades em compreender ou aceitar as implicações da obesidade como uma doença crônica, associada a múltiplos fatores de risco para a saúde, incluindo a doença de diabetes e 0 risco cardiovascular (Prasad et al., 2014). Esses dados são fundamentais para auxiliar a equipe de saúde a conduzir o tratamento 
dos indivíduos obesos de forma que 0 julgamento destes sobre o risco de sua doença seja permeado de ações em relação a autorregulação. Todas essas modificações na capacidade de julgamento exigem uma participação ativa desses indivíduos para superar comorbidades e transformar sua autonomia.

A representação emocional que uma doença crônica como a obesidade gera parece estar inversamente relacionada com a autoeficácia dos indivíduos obesos (Bonsaksen et al., 2012). 0 impacto emocional exerce influência nos determinantes de enfrentamento da doença. Através dos recursos cognitivos e sociais os indivíduos podem monitorar essa resposta emocional à doença e, assim, melhorar a autoeficácia. Nesse entendimento, para ter sucesso através das estratégias de enfrentamento é necessário também mudanças nas crenças de autoeficácia do indivíduo. Desta forma, o indivíduo pode mudar seu comportamento mesmo diante de dificuldades, persistindo em busca de gerir os problemas relacionados à sua doença.

No que se refere a Teoria da Ação Racional, os indivíduos do estudo de Prasad et al. (2014) subestimaram riscos para a saúde. Esses resultados destacam que a opção pela cirurgia bariátrica é vista como benefício maior para restaurar a saúde desses indivíduos, ainda que mudanças comportamentais tenham sido infrutíferas em tratamentos anteriores para redução de fatores de risco.

Conforme o Modelo de Protótipo de Disposição, a vulnerabilidade percebida foi ampliada nos participantes que mantiveram convívio familiar e social com pessoas cujas comorbidades associadas estavam em estágio mais avançado da doença e cujas imagens corporais estavam sujeitas a maior estigma social (Okop, et al., 2016). No entanto, os resultados do estudo sugeriram inadequada percepção de risco da obesidade devido ao desencorajamento social e cultural para perda de peso. Parece que, a avaliação do risco pessoal e a conscientização da doença não é afetada pela imagem corporal e agravamento do quadro clínico de obesidade. Isso demonstra que, quanto maior a vulnerabilidade percebida, maior será a intenção de adotar um comportamento preventivo de saúde. É preciso aprofundar 0 enfrentamento a respeito da doença e da percepção de risco, abordando o problema de forma a intervir para resultados positivos na qualidade de vida.

Com relação ao Modelo Transteórico de Mudança de Comportamento, 0 indivíduo considera a possibilidade de mudança a partir do diagnóstico recebido, ponderando as vantagens e desvantagens de mudar. Nesse sentido, Jumean et al. (2012) verificaram que indivíduos obesos estariam no estágio de contemplação para a mudança. Mesmo com a percepção de risco elevada acerca da doença, os comportamentos habituais alimentares e de atividade física não sofreram alterações. Isso atesta a principal característica do estágio de contemplação que é a ambivalência, em que momentos de ansiedade e dúvida acerca da vontade de mudar enfraquecem a força motivacional do indivíduo. Portanto, a manutenção do peso torna-se 0 maior desafio nesse processo de mudança comportamental.

A intencionalidade não foi preditora de mudança de comportamento nos estudos fundamentados pela Teoria Social Cognitiva (Bonsaksen et al., 2012; Bonsaksen, Fagermoen, \& Lerdal, 2015;). Não se conseguiu atingir resultados significativos de melhora na percepção da doença apenas com os programas educativos. Outros tratamentos associados (farmacoterapia, reeducação alimentar, atividade física regular, cirurgia bariátrica, dentre outros) são necessários para aumentar a preocupação com a saúde. Essa ideia sugere que os indivíduos obesos precisam ser agentes ativos no processo de tomada de decisão, estabelecendo metas e empreendendo a aplicação de estratégias motivacionais, que 
possam sustentar os benefícios alcançados em longo prazo.

Os estudos revisados possuem limitações que precisam ser levadas em consideração ao compreender seus resultados. Comparar indivíduos obesos com indivíduos com doença pulmonar obstrutiva crônica (DPOC), considerada uma doença progressiva e sem cura, foi citado como aspecto limitador das variáveis comparadas (Bonsaksen et al., 2012; Bonsaksen, Fagermoen, \& Lerdal, 2015). As doenças em questão possuem transcurso de progressão e locus de controle diferentes, mesmo que sejam comparadas pelas consequências da dependência do vício de comer e fumar. Embora o programa educativo para os grupos de obesidade e DPOC tenham sido igual, em orientação teórica, a duração com o grupo de obesidade foi maior (9 semanas) do que com o grupo de DPOC (3-5 semanas). Portanto, o programa educativo de obesidade pode ter contribuído para trajetórias mais favoráveis nos indivíduos obesos (Bonsaksen, Lerdal, \& Fagermoen, 2015). No estudo de Ogden e Sidhu (2006) 0 fator limitador foi o pequeno número de participantes e o desenho qualitativo. A percepção de risco sobre a obesidade pode ter sido afetada pelo baixo status sócioeconômico dos participantes, cujas normas culturais valorizam o excesso de peso, como imagem corporal culturalmente desejada e associada a poder econômico (Okop, et al., 2016). A ausência de grupo controle não permitiu inferir relações causais no estudo de Bonsaksen, Lerdal e Fagermoen (2015). 0 desenho de estudo transversal também não permitiu que as relações causais fossem inferidas a partir dos resultados entre a autoeficácia, a atividade física e as percepções individuais (Bonsaksen et al., 2012).

\section{Considerações finais}

A condução clínica da obesidade deve estar amparada em teorias do comportamento em saúde que possam promover seu tratamento com sucesso e auxiliar os indivíduos obesos a terem melhor percepção de risco e percepção da doença de obesidade. Nota-se que há uma dificuldade dos indivíduos de perceberem a obesidade como uma doença crônica que exige esforços contínuos para seu controle. Alguns indivíduos obesos podem até saber que seu excesso de peso é uma ameaça a saúde, mas ao mesmo tempo podem julgar que não é de sua competência modificá-lo quando as causas de sua doença são atribuídas a fatores externos, supostamente, não controláveis pelo próprio indivíduo ou pela influência de normas culturais que desencorajam a perda de peso. Portanto, parece haver dificuldade na avaliação da percepção de risco da obesidade e das suas comorbidades, pois esta não promoveu ações preventivas individuais da doença, nem adoção de comportamentos adequados para melhora do estado de saúde.

A percepção de risco é um construto que auxilia na compreensão da obesidade e pode subsidiar práticas de intervenção de mudança de comportamento alimentar nesses pacientes. No entanto, é importante considerar que outros aspectos, tais como: traços de personalidade, baixa autoestima, ambiente familiar, questões genéticas, normas culturais, também são fatores que intervêm nas atitudes em relação ao comportamento em saúde e na prevenção e promoção da doença da obesidade.

Outros estudos são necessários sobre a temática em questão, especialmente no que tange à avaliação da autorregulação do indivíduo (percepção de risco e percepção da obesidade) e sua relação com a mudança do comportamento alimentar na obesidade. Nesse sentido, estudos que utilizem essas abordagens são importantes para promover melhora na intenção de comportamento e auxiliar no tratamento da doença de obesidade. 


\section{REFERÊNCIAS}

Ajzen, I. (1985). From intentions to actions: A theory of planned behavior. Em J. Kuhl \& J. Beckman (Eds.), Action-control: From cognition to behavior (pp. 11- 39). Heidelberg, Germany: Springer.

Ajzen, I. (1991). The theory of planned behavior. Organizational behavior and human decision processes, 50, 179211. https://doi.org/10.1016/07495978(91)90020-T

Ajzen, I., \& Fishbein, M. (1980). Understanding attitudes and predicting social behavior. Englewood Cliffs, NJ: Prentice-Hall.

Bandura, A. (1977). Self-efficacy: toward a unifying theory of behavioral change. Psychological Review, 84(2), 191-215. https://doi.org/10.1037/0033295X.84.2.191

Bandura, A. (1991). Social cognitive theory of self-regulation. Organizational Behavior and Human Decision Processes, 50, 248-258.

Blixen, C. E., Singh, A., \& Thacker, H. (2006). Values and beliefs about obesity and weight reduction among African American and Caucasian women. Journal of Transcultural Nursing, 17(3), 290-297.

https://doi.org/10.1177/1043659606288 $\underline{375}$

Bonsaksen, T., Fagermoen, M. S., \& Lerdal, A. (2015). Factors associated with selfesteem in persons with morbid obesity and in persons with chronic obstructive pulmonary disease: A cross-sectional study. Psychology, Health \& Medicine, 20(4), 431-442.

https://doi.org/10.1080/13548506.2014. 959529

Bonsaksen, T., Lerdal, A., \& Fagermoen, M. S. (2012). Factors associated with selfefficacy in persons with chronic illness. Scandinavian Journal of Psychology, 53(4), 333-339.

https://doi.org/10.1111/j.14679450.2012.00959.x

Bonsaksen, T., Lerdal, A., \& Fagermoen, M. S. (2015). Trajectories of illness perceptions in persons with chronic illness: An explorative longitudinal study. Journal of Health Psychology, 20(7), 942-953.

https://doi.org/10.1177/1359105313504 $\underline{235}$

Broadbent, E., Petrie, K.J., Main, J., \& Weinman, J. (2006). The brief illness perception questionnaire. Journal of Psychosomatic Research, 60, 631-637. https://doi.org/10.1016/j.jpsychores.200 5.10 .020

Cameron, L., \& Leventhal, H. (2003) Selfregulation, health, and illness: An overview. In L. Cameron \& H. Leventhal (Eds.) The Self-regulation of Health and Illness Behaviour, pp. 1-13.

Crouch, M., \& Leventhal, H. (1997). Are there differences in perceptions of illness across the lifespan? In K. J. Petrie, \& J. Weinman (Eds.). Perceptions of Health and IIIness: Current Research and Applications, (pp. 77-102). Amsterdam.

Diclemente, C. C., Schlundt, B. S., \& Gemmell, L. (2004). Readiness and stages of change in addiction treatment. American Journal on Addictions, 13(2), 103-119.

https://doi.org/10.1080/1055049049043 5777

Falba, T. A., \& Busch, S. H. (2005). Survival expectations of the obese: Is excess mortality reflected in perceptions? Obesity Research, 13(4), 754-761. https://doi.org/10.1038/oby.2005.85

Figueiras, M.J., \& Weinman, J. (2003) Do similar patient and spouse perceptions of myocardial infarction predict recovery? Psychology and Health, 18(2), 201-216.

https://doi.org/10.1080/0887044021000 057266

Fishbein, M., \& Aizen, I. (1975). Belief, attitude, intention, and behavior: An introduction to theory and research. Reading, MA: Addison-Wesley.

French, D. P., Marteau, T. M., Senior, V., \& Weinman, J. (2000). Perceptions of multiple risk factors for heart attacks. 
Psychological Reports, 87(2), 681-687. https://doi.org/10.2466/pr0.2000.87.2.6 81

Furze, G., Roebuck, A., Bull, P., Lewin, R. J., \& Thompson, D. R. (2002). A comparison of the illness beliefs of people with angina and their peers: a questionnaire study. BMC Cardiovascular Disorders, 2, 4. https://doi.org/10.1186/1471-2261-2-4

Hilbert, A., Dierk, J. M., Conradt, M., Schlumberger, P., Hinney, A., Hebebrand, J., \& Rief, W. (2009). Causal attributions of obese men and women in genetic testing: implications of genetic/biological attributions. Psychology Health, 24(7), 749-761. https://doi.org/10.1080/0887044080194 $\underline{7787}$

Holmen, J., Midthjell, K., Krüger, O., Langhammer, A., Holmen, T.L., Bratberg, G.L., \& Lund-Larsen, P. G. (2003). The Nord-Trondelag health study 1995-1997 (HUNT-2): Objectives, contents, methods, and participation. Norsk Epidemiologi, 13, 19-32. https://pdfs.semanticscholar.org/a18f/8 4bb399dfeaede1619e0ac80dabf18711 $8 \mathrm{c} 0 . \mathrm{pdf}$

Johnson, P., Fallon, E. A., Harris, B. S., \& Burton, B. (2013). Body satisfaction is associated with Transtheoretical Model constructs for physical activity behavior change. Body Image, 10(2), 163-174. 10.1016/j.bodyim.2012.12.002

Johnson, S. S., Driskell, M. M., Johnson, J. L., Prochaska, J. M., Zwick, W., \& Prochaska, J. O. (2006). Efficacy of a transtheoretical model-based expert system for antihypertensive adherence. Disease Management, 9(5), 291-301. https://doi.org/10.1089/dis.2006.9.291

Jumean, M. F., Korenfeld, Y., Somers, V. K., Vickers, K. S., Thomas, R. J., \& LopezJimenez, F. (2012). Impact of diagnosing metabolic syndrome on risk perception. American Journal Health Behavior, 36(4), 522-532.

https://doi.org/10.5993/AJHB.36.4.9
Juszczyk, D., Gillison, F. B., \& Dean, S. (2014). Can media images of obese people undermine health messages? An experimental study of visual representation and risk perception. European Journal of Public Health, 24(6), 930-935.

https://doi.org/10.1093/eurpub/cku064

Kaptein, A. A., Van Korlaar, I. M., Cameron, L. D., Vossen, C. Y., Van der Meer, F. J., \& Rosendaal, F. R. (2007). Using the common-sense model to predict risk perception and disease-related worry in individuals at increased risk for venous thrombosis. Health Psychology, 26(6), 807-812. https://doi.org/10.1037/02786133.26 .6 .807

Lerdal, A., Andenaes, R., Bjornsborg, E., Bonsaksen, T., Borge, L., Christiansen, B., \& Fagermoen, M. S. (2011). Personal factors associated with healthrelated quality of life in persons with morbid obesity on treatment waiting lists in Norway. Quality of Life Research, 20(8), 1187-1196.

https://doi.org/10.1007/s11136-0119865-z

Leventhal, H., Brissete, I., \& Leventhal, E. (2003). The common-sense model of self-regulation of health and illness. In $\mathrm{L}$. Cameron, \& H. Leventhal (Eds.), The self-regulation of health and behaviour, (pp.42-65). London: Routledge.

Leventhal, H., Nerenz, D., \& Steele, D. (1984). Illness representations and coping with health threats. In Baum, A., Singer, J. (Eds.). Handbook of psychology and health (221-252). Erlbaum: New York.

Mastellos, N., Gunn, L. H., Felix, L. M., Car, J., \& Majeed, A. (2014). Transtheoretical model stages of change for dietary and physical exercise modification in weight loss management for overweight and obese adults. Cochrane Database of Systematic Reviews, 2, CD008066. https://doi.org/10.1002/14651858.CD00 8066.pub3

Moher, D., Liberati, A., Tetzlaff, J., Altman, D. G., \& Group, P. (2009). Reprint- 
preferred reporting items for systematic reviews and meta-analyses: the PRISMA statement. Physical Therapy, 89(9), 873-880.

https://doi.org/10.1093/ptj/89.9.873

Noria, S. F., \& Grantcharov, T. (2013). Biological effects of bariatric surgery on obesity-related comorbidities. Canadian Journal of Surgery, 56(1), 47-57. https://doi.org/10.1503/cjs.036111

Ogden, J., \& Sidhu, S. (2006). Adherence, behavior change, and visualization: a qualitative study of the experiences of taking an obesity medication. Journal Psychosomatic Research, 61(4), 545552. 10.1016/j.jpsychores.2006.04.017

Okop, K. J., Mukumbang, F. C., Mathole, T., Levitt, N. \& Puoane, T. (2016). Perceptions of body size, obesity threat and the willingness to lose weight among black South African adults: a qualitative study. BMC Public Health, 16 (365). https://doi.org/10.1186/s12889016-3028-7

Park, S., Cochrane, B. B., Koh, S. B., \& Chung, C. (2011). Comparison of breast cancer risk estimations, risk perception, and screening behaviors in obese rural Korean women. Oncology Nursing Forum, 38(6), E394-401. https://doi.org/10.1188/11.ONF.E394E401

Parschau, L., Barz, M., Richert, J., Knoll, N., Lippke, S., \& Schwarzer, R. (2014). Physical activity among adults with obesity: testing the Health Action Process Approach. Rehabilitation Psychology, 59(1), 42-49. https://doi.org/10.1037/a0035290

Peters, E., Slovic, P., Hibbard, J. H., \& Tusler, M. (2006). Why worry? Worry, risk perceptions, and willingness to act to reduce medical errors. Health Psychology, 25(2), 144-152.

https://doi.org/10.1037/02786133.25.2.144

Prasad, C., Batsis, J. A., Lopez-Jimenez, F., Clark, M. M., Somers, V. K., Sarr, M. G., \& Collazo-Clavell, M. L. (2014). Risk perception of obesity and bariatric surgery in patients seeking treatment for obesity. European Journal of Preventive Cardiology, 21(6), 692-703.

https://doi.org/10.1177/2047487312466 $\underline{904}$

Prochaska J.O. (1979). Systems of psychotherapy: a transtheoretical analysis. Homewood, IL: Dorsey Press.

Prochaska J. O., \& DiClemente, C.C. (1982). Trans-theoretical therapy - toward a more integrative model of change. Psychotherapy: Theory, Research and Practice, 19(3), 276-288.

Prochaska, J. O., \& Velicer, W.F. (1997). The Transtheoretical Model of health behavior change. American Journal of Health Promotion, 12, 38-48.

https://doi.org/10.4278/0890-1171$\underline{12.1 .38}$

Prochaska, J. O., Butterworth, S., Redding, C. A., Burden, V., Perrin, N., Leo, M., \& Prochaska, J. M. (2008). Initial efficacy of MI, TTM tailoring and HRI's with multiple behaviors for employee health promotion. Preventive Medicine, 46(3), 226-231.

https://doi.org/10.1016/j.ypmed.2007.11 .007

Prochaska, J. O., DiClemente, C. C., \& Norcross, J. C. (1992). In search of how people change: Applications to addictive behavior. American Psychologist, 47, 1102-1114.

https://doi.org/10.3109/1088460930914 9692

Radtke, T., Kaklamanou, D., Scholz, U., Hornung, R., \& Armitage, C. J. (2014). Are diet-specific compensatory health beliefs predictive of dieting intentions and behaviour? Appetite, 76, 36-43. https://doi.org/10.1016/j.appet.2014.01. $\underline{014}$

Rivis A, Sheeran P, Armitage CJ (2006). Augmenting the theory of planned behaviour with the prototype/willingness model: predictive validity of actor versus abstainer prototypes for adolescents' health-protective and healthrisk intentions. British Journal of Health Psychology, 11(Pt 3),483-500. 
https://doi.org/10.1348/135910705X70327

Rosenberg, M. (1965). Society and the adolescent self-image. Princeton, NJ: Princeton University Press.

Schwarzer, R., \& Jerusalem, M. (1995). Generalized self-efficacy scale. In J. Weinman, S. Wright, \& M. Johnston (Eds.), Measures in health psychology: A user's portfolio (pp. 35-37). Windsor, UK: Nfer-Nelson.

Siegrist, M., \& Cvetkovich, G. (2000). Perception of hazards: the role of social trust and knowledge. Risk Analysis, 20(5),

713-719.

https://doi.org/10.1111/02724332.205064

Simpson, J., Lekwuwa, G., \& Crawford, T. (2013). Illness beliefs and psychological outcome in people with Parkinsons disease. Chronic Illness, 9, 165-176. https://doi.org/10.1177/1742395313478 219

Smith JA, \& Osborn M. (2003). Interpretative phenomenological analysis. In J.A. Smith (Ed). Qualitative psychology. Sage, pp. 51- 80.

Sjöberg, L. (1998). Worry and risk perception. Risk Analysis, 18(1), 85-93. https://doi.org/10.1111/j.15396924.1998.tb00918.x

Slovic, P. (1987). Perception of risk. Science, 236(4799), 280-285.

https://doi.org/10.1126/science.3563507

Slovic, P., Finucane, M. L., Peters, E., \& MacGregor, D. G. (2004). Risk as analysis and risk as feelings: some thoughts about affect, reason, risk, and rationality. Risk Analysis, 24(2), 311322. https://doi.org/10.1111/j.02724332.2004.00433.x

Teede, H. J., Lombard, C., \& Deeks, A. A. (2010). Obesity, metabolic complications and the menopause: an opportunity for prevention. Climacteric, 13(3), 203-209.

https://doi.org/10.3109/1369713090329 $\underline{6909}$

Wee, C. C., Davis, R. B., \& Phillips, R. S. (2005). Stage of readiness to control weight and adopt weight control behaviors in primary care. Journal of General Internal Medicine, 20(5), 410415. https://doi.org/10.1111/j.15251497.2005.0074.x

Wilson P.W., D'Agostino R.B., Levy D., Belanger A.M., Silbershatz H., \& Kannel, WB. (1998). Prediction of coronary heart disease using risk factor categories. Circulation, 97, 1837-1847. https://doi.org/10.1161/01.CIR.97.18.18 $\underline{37}$

Winter, J., \& Wuppermann, A. (2014). Do they know what is at risk? Health risk perception among the obese. Health Economy, 23(5), 564-585. https://doi.org/10.1002/hec.2933

Yamada-Goto, N., Katsuura G., \& Nakao K. (2013). A Novel approach to obesity from mental Function. Journal of Obesity \& Weigth Loss Therapy, 3(167). https://doi.org/10.4172/21657904.1000168

Todos los trabajos publicados en Revista Salud \& Sociedad (ISSNe:0718-7475) están sujetos a una licencia Creative Commons Reconocimiento 4.0 Internacional

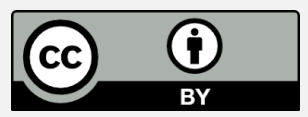




\section{RESUMO}

ANTECEDENTES: A obesidade tem sido descrita como uma doença crônica, multifatorial e de caráter evolutivo colocando em risco a vida dos indivíduos. OBJETIV0: 0 objetivo do artigo foi realizar uma revisão sistemática sobre percepção da doença e de risco na obesidade, entre janeiro de 2004 e janeiro de 2018. MÉTODO: Foram pesquisadas nas bases de dados Lilacs, SciELO, MEDLINE, PubMed, PsycINFO e Web of Science, estudos que investigavam a percepção da doença e de risco da obesidade tendo como base teorias comportamentais e sociais que ajudam a compreender por que pessoas adultas se engajam em comportamentos de risco. RESULTADOS: A partir de 577 referências, sete estudos preencheram os critérios de inclusão. Percebeu-se que indivíduos obesos têm dificuldade de entender sua doença como uma doença crônica que exige esforços contínuos para seu controle. A avaliação da percepção de risco da obesidade não promoveu ações preventivas individuais da doença, nem adoção de comportamentos adequados para melhora do estado de saúde. CONCLUSÃO: A percepção de risco é um construto que auxilia na compreensão da obesidade e pode subsidiar práticas de intervenção de mudança de comportamento alimentar nos pacientes.

PALAVRAS-CHAVE: Percep̧̧̃̃o de risco, Percepção da doença, Crenças, Obesidade.

\section{RESUMEN}

ANTECEDENTES: La obesidad se ha descrito como una enfermedad crónica, multifactorial y evolutiva que pone en riesgo la vida de las personas. OBJETIVO: El objetivo de este estudio fue realizar una revisión sistemática de literatura sobre la percepción de la enfermedad y el riesgo de la obesidad entre enero de 2004 y enero de 2018. MÉTODO: Buscamos en las bases de datos Lilacs, SciELO, MEDLINE, PubMed, PsycINFO y Web of Science, estudios que investigan la percepción de la enfermedad y el riesgo de obesidad basado en teorías conductuales y sociales que ayudan a entender por qué los adultos se involucran en conductas de riesgo. RESULTADOS: A partir de 577 referencias, siete estudios cumplieron con los criterios de inclusión. Identificamos que los individuos obesos tienen dificultad para entender su enfermedad como una crónica que requiere esfuerzos continuos para controlarlo. La evaluación de la percepción del riesgo de obesidad no promocionó las acciones preventivas individuales de la enfermedad ni la adopción de comportamientos adecuados para mejorar el estado de salud. CONCLUSIÓN: La percepción de riesgos es una construcción que ayuda a entender la obesidad y puede subsidiar las prácticas de intervención de cambio de comportamiento en los pacientes.

PALABRAS CLAVE: Percepción de riesgo, Percepción de enfermedad, Creencias de enfermedad, Obesidad. 